\title{
The Effect of Monocular Blur on the Binocular Visual Field
}

\author{
Fusako Fujimura ${ }^{{ }^{*}}$, Nobuyuki Shoji ${ }^{2}$ \\ ${ }^{1}$ Department of Rehabilitation, Orthoptics, and Visual Science Course, School of Allied Health Sciences, Kitasato \\ University, Kanagawa, Japan \\ ${ }^{2}$ Department of Ophthalmology, School of Medicine, Kitasato University, Kanagawa, Japan \\ Email: ^f-fujimu@kitasato-u.ac.jp, nshoji@ahs.kitasato-u.ac.jp
}

How to cite this paper: Fujimura, F. and Shoji, N. (2018) The Effect of Monocular Blur on the Binocular Visual Field. Open Journal of Ophthalmology, 8, 12-17. https://doi.org/10.4236/ojoph.2018.81002

Received: January 4, 2018

Accepted: February 2, 2018

Published: February 5, 2018

Copyright $\odot 2018$ by authors and Scientific Research Publishing Inc. This work is licensed under the Creative Commons Attribution International License (CC BY 4.0).

http://creativecommons.org/licenses/by/4.0/

\begin{abstract}
Purpose/Aim: We aimed to investigate the effect of monocular blur on the binocular visual field. Materials and Methods: A total of 13 healthy young volunteers participated in this study. The mean subjective refractive error of the dominant eye $(\mathrm{DE})$ was $-3.33 \pm 1.65 \mathrm{D}$, and the non-dominant eye (NDE) was $-3.15 \pm 2.84 \mathrm{D}$. The $\mathrm{DE}$ was determined by using the hole-in-the-card test. The visual field was examined by the Humphrey Visual Field Analyzer using the 30-2 SITA Standard program. The visual field was measured while wearing soft contact lens under three conditions; (1) both eyes: near vision correction; (2) DE: near vision correction +3.00D added, NDE: near vision correction; and (3) DE: near vision correction, NDE: near vision correction +3.00D added. The foveal threshold, mean deviation (MD), and pattern standard deviation (PSD) values were investigated. Results: The foveal threshold value $(\mathrm{dB})$ at (1), (2), and (3) was $41.2,37.8$, and 38.1 , respectively. The values at (2) and (3) were both significantly lower than that at (1) $(p<0.0001 ; p=$ $0.0003)$. The MD value (dB) at (1), (2), and (3) was $1.67,0.19$, and 0.51 , respectively; the values at (2) and (3) were both significantly lower than that at (1) $(p=0.0012 ; p=0.0118)$. The PSD value ( $\mathrm{dB})$ at (1), (2), and (3) was 1.36 , 1.55 , and 1.47, respectively. A significant difference in (1), (2), and (3) was not found. Conclusion: These results suggest that monocular blur reduced the sensitivity within the binocular visual field.
\end{abstract}

\section{Keywords}

Binocular Visual Field, Monocular Blur, Binocular Inhibition, Eye Dominance, Humphrey Visual Field Analyze

\section{Background}

In general, visual field is measured under monocular view in clinics. However, in 
daily life, visual information is managed with both eyes open. To consider the vision quality for a patient's daily life, evaluating the visual field under binocular open view is important. In cases of monocular visual field abnormality, visual field abnormality is complemented by the normal visual field of the fellow eye under binocular open view condition. Hence, awareness of the visual disturbance is difficult for the patient. In contrast, many patients with monocular cataract also complain under binocular open view condition. However, there are fewer studies on the effect of monocular blur on the binocular visual field result.

In this study, we created a monocular pseudo blur in this study, and investigated the effect of monocular blur within the binocular visual field.

\section{Materials and Methods}

We examined 13 healthy young volunteers ( 1 man and 12 women; mean age, 21 [range: 20 - 22 years]) in this study, and have publicly invited subjects by poster postingat School of Allied Health Sciences, Kitasato University. All subjects underwent ophthalmology examination. Subjects were excluded from the study if they had any ophthalmologic disease except refractive error. The mean subjective refractive error (spherical equivalent value, $\mathrm{SE}$ ) of the dominant eye (DE) was $-3.33 \pm 1.65 \mathrm{D}$, and the $\mathrm{SE}$ of the non-dominant eye (NDE) was $-3.15 \pm$ $2.84 \mathrm{D}$. The dominant eye was determined by using the hole-in-the-card test. The visual field was examined by using the Humphrey Visual Field Analyzer 740 (Carl Zeiss, Dublin, CA) using Swedish Interactive Threshold Algorithm 30-2 SITA Standard. We measured the visual field while wearing soft contact lens under the following three conditions:

1) Both eyes: near vision correction (both near conditions)

2) $\mathrm{DE}$ : near vision correction $+3.00 \mathrm{D}$ addition/NDE: near vision correction (DE blur condition)

3) $\mathrm{DE}$ : near vision correction/NDE: near vision correction $+3.00 \mathrm{D}$ addition (NDE blur condition)

The measurement of these three conditions was randomly performed. We placed the center of both eyes that coincides with the center of the fixation monitor during the measurement. Subsequently, we compared the foveal threshold, mean deviation (MD), and pattern standard deviation (PSD) values between the measurements of the three conditions.

The study protocol was approved by the Institutional Review Board at the School of Allied Health Sciences of Kitasato University (number 2015-020) and followed the tenets of the Declaration of Helsinki. Informed consent was obtained from all patients after explanation of the nature and possible consequences of the study.

\section{Statistical Analysis}

Statistical analysis was performed by using IBM SPSS statistics software (version 23.0; IBM Corporation, Armonk, NY, USA). The Tukeytest was used to compare 
the foveal threshold, MD, and PSD values between the measurements of the three conditions. The level of statistical significance was set at a $p$-value less than 0.05 .

\section{Results}

Figure 1 shows the foveal threshold values under three conditions (both near, DE blur, and NDE blur conditions). The foveal threshold values were $41.2 \pm 1.2$ $\mathrm{dB}, 37.8 \pm 1.7 \mathrm{~dB}$, and $38.1 \pm 2.2 \mathrm{~dB}$, respectively. The foveal threshold values under DE blur and NDE blur conditions were significantly different from both near condition values $(p<0.0001 ; p=0.0003)$.

Figure 2 shows the $\mathrm{MD}$ values under three conditions (both near, DE blur, and NDE blur conditions). The MD values were $1.67 \pm 1.00 \mathrm{~dB}, 0.19 \pm 0.99 \mathrm{~dB}$, and $0.51 \pm 0.94 \mathrm{~dB}$, respectively. The MD values under DE blur and NDE blur conditions were significantly different from both near condition values ( $p=$ 0.0012; $p=0.0118$ ). No significant differences were found between DE blur and NDE blur conditions in the foveal threshold and MD values. The values for the DE blur condition tended to be lower than those for the NDE blur condition. The PSD values were $1.36 \pm 0.19 \mathrm{~dB}, 1.55 \pm 0.28 \mathrm{~dB}$, and $1.47 \pm 0.18 \mathrm{~dB}$, respectively. No significant differences were found between three conditions (Figure 3). The values for the DE blur condition tended to be higher than those for the NDE blur condition.

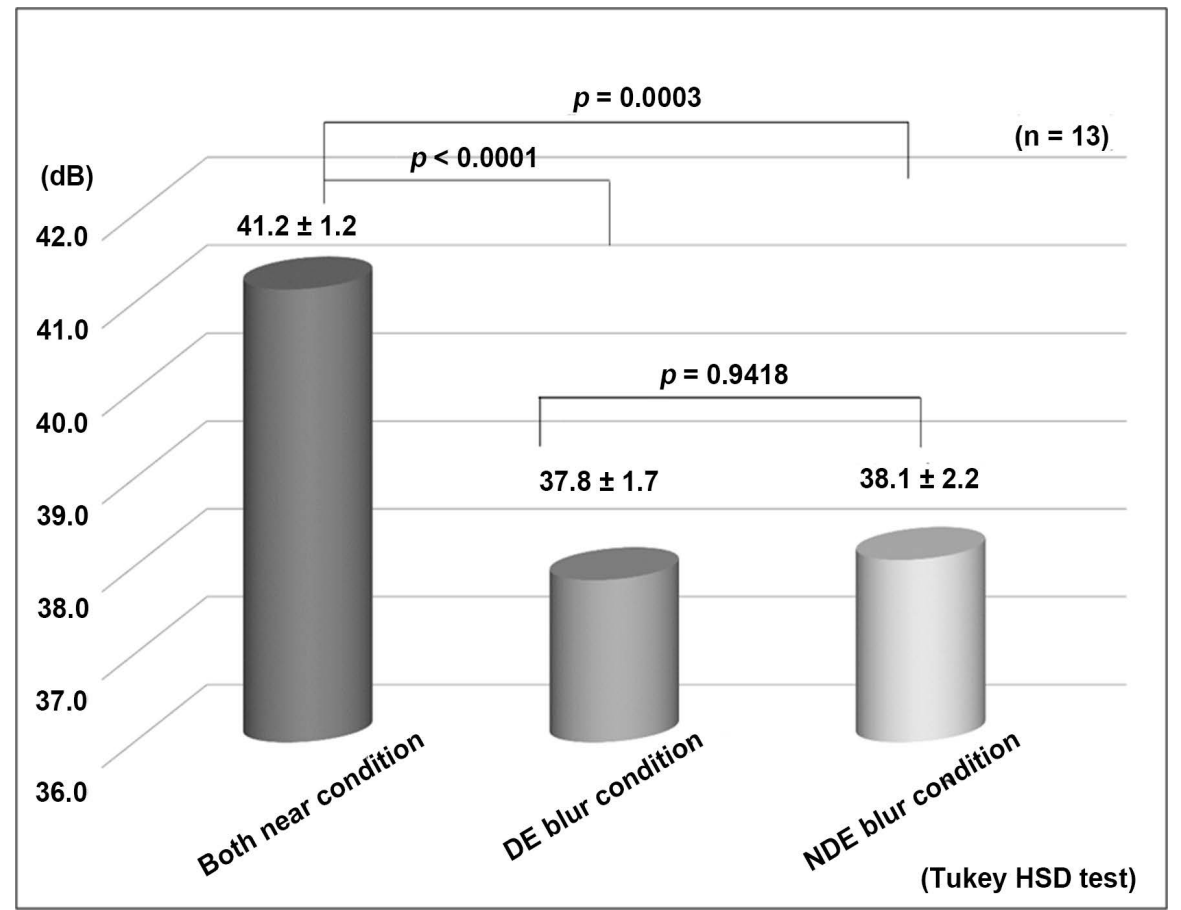

Figure 1. Foveal threshold values. DE: dominant eye; NDE: non-dominant eye; Both near condition: Both eyes were corrected for near vision. DE blur condition: DE was added + 3.00D to near vision correction/NDE was corrected for near vision; NDE blur condition: $\mathrm{DE}$ was corrected for near vision /NDE was added +3.00D to near vision correction. 


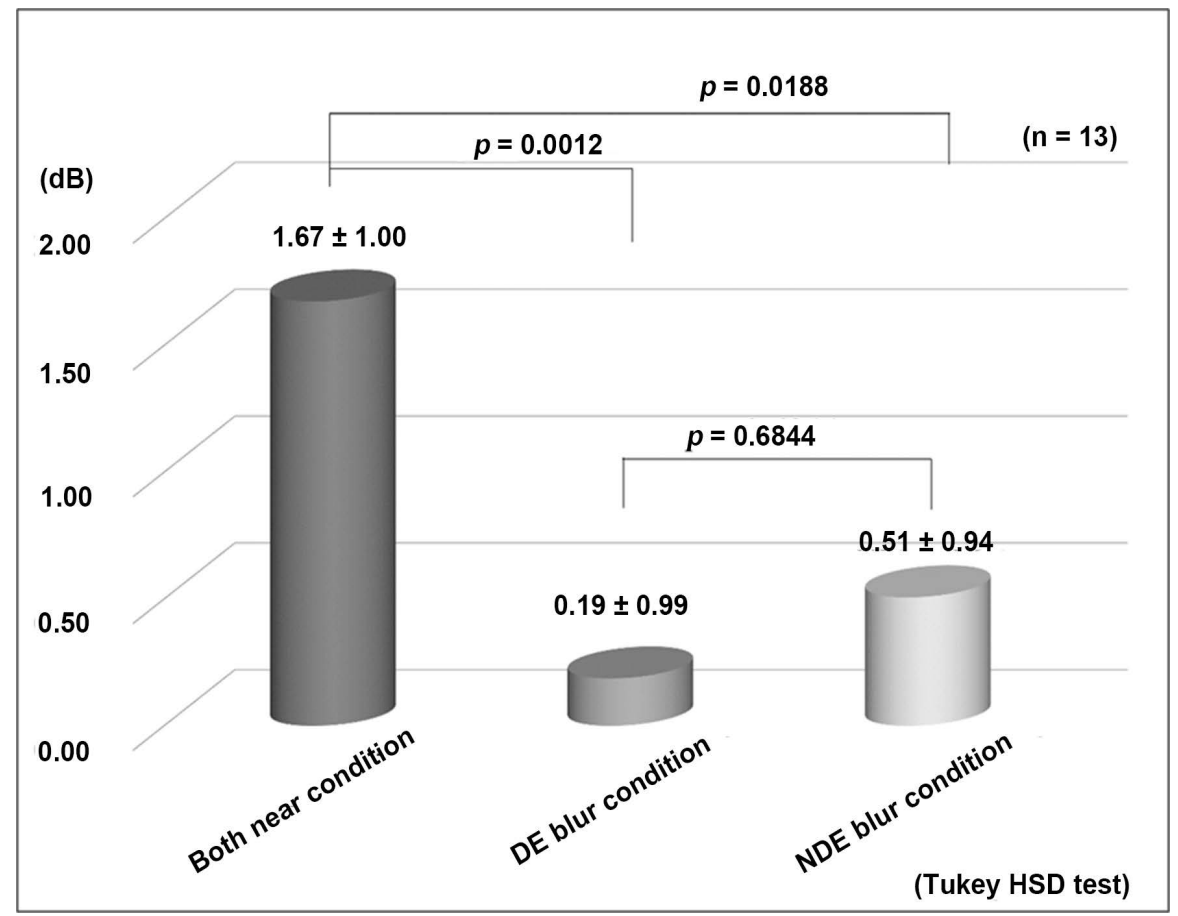

Figure 2. MD values. DE: dominant eye; NDE: non-dominant eye; Both near condition: Both eyes were corrected for near vision. DE: blur condition: DE was added $+3.00 \mathrm{D}$ to near vision correction/NDE was corrected for near vision; NDE blur condition: DE was corrected for near vision/NDE was added $+3.00 \mathrm{D}$ to near vision correction.

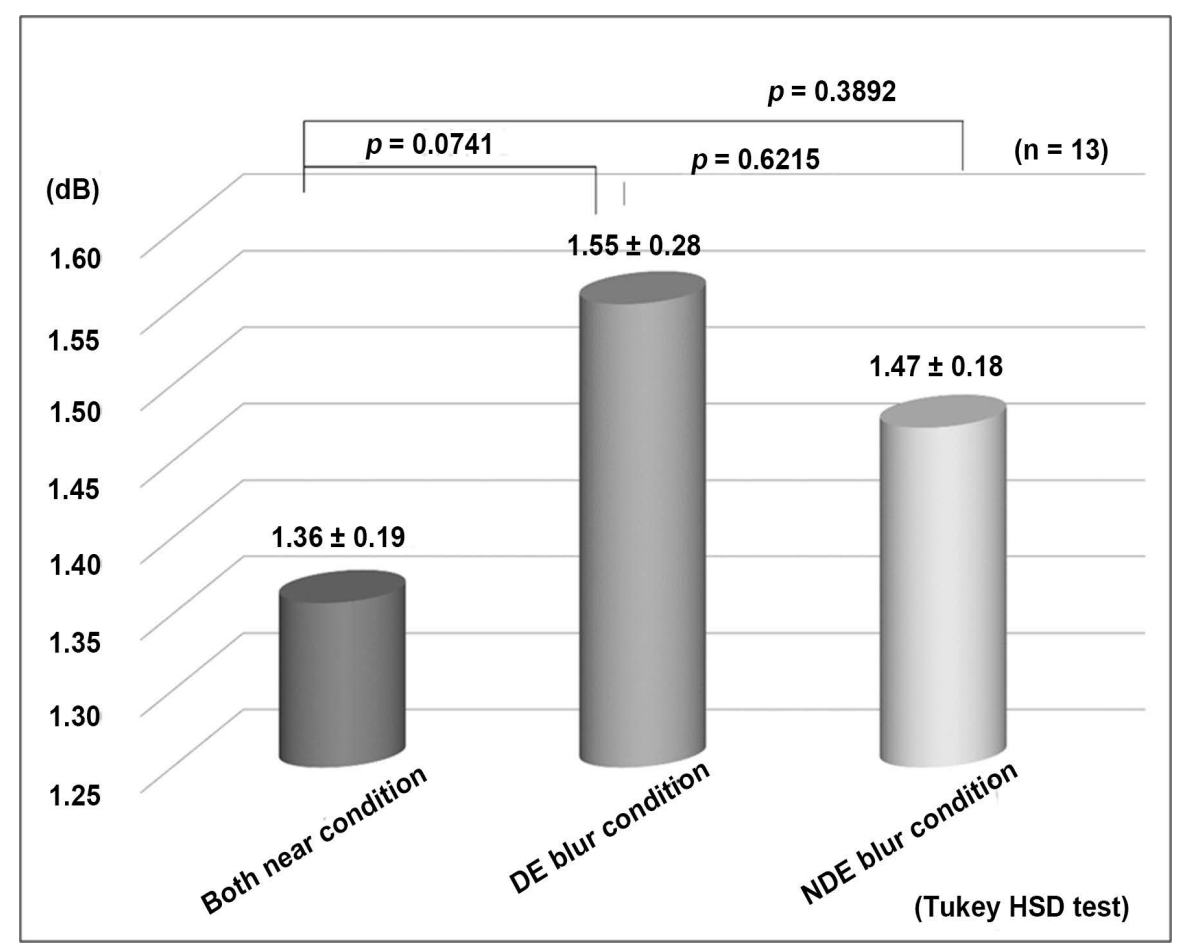

Figure 3. PSD values. DE: dominant eye; NDE: non-dominant eye; Both near condition: Both eyes were corrected for near vision. DE: blur condition: DE was added $+3.00 \mathrm{D}$ to near vision correction/NDE was corrected for near vision; NDE blur condition: DE was corrected for near vision/NDE was added +3.00D to near vision correction. 


\section{Discussion}

In this study, significant differences were found between both near conditions and DE blur, NDE blur conditions in the foveal threshold values and MD values.

Pardhan et al. [1] reported that with increasing difference in monocular illuminance, the binocular response decreased steadily until it reached a level below the monocular illuminance, and this perceptual phenomenon was defined as binocular inhibition. Gilchrist et al. [2] [3] reported that binocular psychometric functions were also measured when the sensitivity of one eye was decreased by means of a 1.0 neutral density filter. They found that binocular detectability in this case was reduced to below that of the better eye. This binocular inhibition was seen in all subjects. In this study, binocular inhibition was suggested to possibly occur by monocular blur as the threshold decrease in the binocular visual field measurement. In contrast, no differences were found between the PSD values of the three conditions. The PSD values indicate the presence of irregular visual field shapes and local scotoma. Local reduction in sensitivity was not observed under this measurement, and we used the convex lens to blur one eye; hence, it was inferred to have no significant difference in the PSD value.

Based on these results, no significant difference was found in the foveal threshold, the MD, and PSD values under DE blur and NDE blur conditions, and the values under DE blur condition had a greater tendency to indicate deterioration.

Under natural viewing conditions, humans demonstrate ocular dominance, and this feature is an important aspect of binocular vision. Usually, the dominant eye plays a controlling role in binocular vision [4] [5] [6] [7] [8]. Handa et al. reported that in the center 10-2 threshold measurement, the threshold decreased in the binocular open, non-dominant eye occlusion, and dominant eye occlusion conditions. They reported the occurrence of a strong interference by the dominant eye occlusion at the time of the visual field measurement. DE blur condition was suggested to affect visual field sensitivity under binocular open view.

In this study, a convex lens of $3.00 \mathrm{D}$ was added to create the pseudo monocular blur. It was inferred that not to be the result of reflecting the chief complaint against the natural viewing of patients because this situation is different from the blur encountered in conditions such as cataracts. This may have resulted in aniseikonia by adding the convex lens to the eye, and this situation possibly affects the fusion in binocular vision. Moreover, the perimeter used in this study has not been designed for measuring the visual field under binocular open view. Fixation monitoring, eye position, and fusion situation during the measurement were not observed; hence, these can be cited as problems. We considered examining and improving the testing method for these points necessary in the future.

\section{Conclusion}

In this study, we investigated the effect of monocular blur to the binocular visual 
field by monocular pseudo blur. The sensitivity decrease within the binocular visual field was suggested to be influenced by the monocular blur.

\section{Availability of Data and Materials}

Data can be shared upon request.

\section{Authors' Contributions}

Conception and design of the study (FF, NS); analysis and interpretation (FF); writing the article (FF); critical revision of the article (FF, NS); data collection (FF); administrative, technical or logistic support (NS). All authors read and approved the final manuscript.

\section{Consent for Publication}

A written informed consent to publish study participant's data was sought along with the consent for participation into the study.

\section{Ethics Approval and Consent to Participate}

This study was approved by the Institutional Review Board at School of Allied Health Sciences, Kitasato University (number 2015-020) and followed the tenets of the Declaration of Helsinki.

\section{References}

[1] Pardhan, S., Gilchrist, J., Douthwaite, W. and Yap, M. (1990) Binocular Inhibition: Psychophysical and Electrophysiological Evidence. Optometry and Vision Science, 67, 688-691. https://doi.org/10.1097/00006324-199009000-00006

[2] Gilchrist, J. and McIver, C. (1985) Fechner's Paradox in Binocular Contrast Sensitivity. Vision Research, 25, 609-613. https://doi.org/10.1016/0042-6989(85)90167-1

[3] Gilchrist, J. and Pardhan, S. (1987) Binocular Contrast Detection with Unequal Monocular Illuminance. Ophthalmic and Physiological Optics, 7, 373-377. https://doi.org/10.1111/j.1475-1313.1987.tb00763.x

[4] Reiss, M.R. (1997) Ocular Dominance: Some Family Data. Laterality, 2, 7-16. https://doi.org/10.1080/713754254

[5] Sengpiel, F., Blakemore, C., Kind, P.C. and Harrad, R. (1994) Interocular Suppression in the Visual Cortex of Strabismic Cats. Journal of Neuroscience, 14, 6855-6871.

[6] Horng, J.L., Semmlow, J.L., Hung, G.K. and Ciuffreda, K.J. (1998) Dynamic Asymmetries in Disparity Convergence Eye Movements. Vision Research, 38, 2761-2768. https://doi.org/10.1016/S0042-6989(97)00453-7

[7] Kawata, H. and Ohtsuka, K. (2001) Dynamic Asymmetries in Convergence Eye Movements under Natural Viewing Conditions. Japanese Journal of Ophthalmology, 45, 437-444. https://doi.org/10.1016/S0021-5155(01)00405-1

[8] Handa, T. and Shoji, N. (2006) Effect of Ocular Dominance in the Visual Field Test. Atarashi Gannka, 23, 1029-1030. 